\title{
Remoción de cromo de efluentes de la industria curtiembre mediante electrodiálisis
}

\begin{abstract}
RESUMEN
Las sales de cromo son reactivos químicos muy utilizados en la industria de curtiembre porque son eficientes para curtir la piel animal, sin embargo, estas sales no se fijan totalmente, por lo que, sus remanentes van a parar a los efluentes, impactando gravemente al medioambiente. El objetivo de este estudio fue remover el cromo (VI) y cromo total de un efluente de curtiembre utilizando electrodiálisis, para ello, se caracterizó una muestra de un efluente de una planta piloto de curtiembre y se preparó una solución sintética en base a ésta, utilizando una sal de cromo para curtiembres de grado industrial, luego, se determinó la corriente límite y se realizó pruebas de electrodiálisis con el 80,100 y $120 \%$ de la corriente límite. Los resultados demostraron que el mayor porcentaje de extracción y el menor consumo de energía eléctrica fue al $80 \%$ de la corriente límite.
\end{abstract}

Palabras-claves: Curtiembre; Cromo; Electrodiálisis.

REMOVAL OF CHROMIUM FROM EFFLUENTS OF TANNERY INDUSTRY BY ELECTRODIALYSIS

\section{ABSTRACT}

Chromium salts are chemical reagents widely used in the tannery industry because they are efficient for tanning animal skin, however, these salts are not completely fixed, so their remnants go to their effluents, seriously impacting the environment. The objective of this study was to remove chromium (VI) and total chromium from a tannery effluent using electrodialysis, for this purpose, a sample of an effluent from a tannery pilot plant was characterized and then a synthetic solution was prepared based on this, using an industrial grade chrome salt for tannery, then the limiting current was determined and electrodialysis tests were carried out with the 80,100 and $120 \%$ of the limiting current. The results showed that the highest percentage of extraction and the lowest electricity consumption was $80 \%$ of the limiting current.

Keywords: Tannery; Chromium; Electrodialysis.

\section{INTRODUCCIÓN}

El sulfato básico de cromo es el agente químico más utilizado en la industria de curtiembre. Su eficiencia es mayor que utilizar agentes vegetales de curtición, sin embargo, el cromo no se fija por completo en la piel animal, por eso, los remanentes son desechados en sus efluentes (Chávez, 2010), impactando fuertemente al medio ambiente. El cromo que hace referencia este estudio son cromo (VI) y cromo totales, estos últimos son parámetros de efluentes líquidos al cuerpo receptor (D.S. $\mathrm{N}^{\circ}$ 003-2002-PRODUCE) y descargas de aguas residuales no domésticas en el sistema de alcantarillado sanitario (D.S. $\mathrm{N}^{\circ}$ 021-2009-VIVIENDA) para la actividad industrial de curtiembre en el Perú.

Existen diversos procesos para remover el cromo, como: precipitación, intercambio iónico, materiales adsorbentes, biomateriales, biotecnología y electrocoagulación (Chávez, 2010). Otro proceso de remoción basado en membranas es la electrodiálisis (Lambert et al., 2006).

La electrodiálisis es un proceso de separación con electromembrana, se basa en la selectividad de las membranas de intercambio iónico. Estas membranas contienen grupos funcionales con cargas eléctricas fijas en la matriz polimérica, de modo que, permiten la permeación de los iones de carga opuesta en una solución, bajo la influencia de un campo eléctrico continuo (Koter y Warszawski, 2000). La electrodiálisis se aplica para extraer especies químicas iónicas a partir de soluciones acuosas. Por lo general, la alimentación es una solución que contiene iones que al ser tratadas mediante electrodiálisis se obtienen dos soluciones como productos: diluida y concentrada (Martí, 2014). A escala industrial la electrodiálisis se usa para desalar aguas salobres, tratar aguas residuales, producir sal de mesa y desmineralizar el agua de alimentación de la caldera y productos alimenticios (Strathmann, 2010).

\footnotetext{
M.Sc. en Ingeniería Química FIQT-UNI, apilco@uni.edu.pe, Docente FIQT-UNI

2 Mg. en Gestión de Operaciones y Servicios Logísticos FII-UNMSM, tinocofelix@gmail.com, Consultor independiente

3 Ingeniero Químico FIQT-UNI, pedro.mamani.p@gmail.com, Consultor independiente

4 Ingeniero Químico FIQT-UNI, gyallicoa@hotmail.com, Consultor independiente
} 
La corriente límite es un parámetro importante para una prueba de electrodiálisis y se determina experimentalmente a partir de la curva corriente-voltaje. La corriente límite está relacionada con la eficiencia para utilizar la corriente eléctrica suministrada al módulo de electrodiálisis, por eso, se determina como paso previo antes de una prueba. Una guía que hace referencia al diseño de módulos de electrodiálisis respecto a la corriente límite es Ochoa (1996), que indica que este parámetro debe ser del orden del $70 \%$ de la corriente límite.

La curva de corriente-voltaje está compuesta por tres regiones (Praneeth et al., 2014). La primera zona muestra una relación lineal entre la corriente y el voltaje (región óhmica). En la segunda zona la corriente varía muy ligeramente con el voltaje (meseta), correspondiendo a la llamada corriente límite; es aquí en donde se inicia la hidrólisis del agua generándose nuevas especies $\left(\mathrm{H}^{+}\right.$y $\left.\mathrm{OH}^{-}\right)$, quienes logran disminuir la eficiencia de la corriente. En la tercera zona, la corriente aumenta nuevamente con el voltaje aplicado, a esta zona también se le conoce como región de electroconvección.

En ocasiones, la región de la corriente límite no se puede apreciar experimentalmente, por eso, existen diversos procedimientos para determinarlo. Cowan y Brown (1959) determinaron la corriente límite a partir de los datos experimentales de una curva de corriente-voltaje, realizando una segunda curva (Resistencia - Inversa de la corriente) y considerando el valor mínimo local. Ponce de León y Field (2000) demostraron que la corriente límite se puede determinar a partir de la segunda curva (Resistencia - Inversa de la corriente), conociendo los valores del máximo y mínimo locales.

Existen estudios sobre la remoción del cromo con electrodiálisis, Raghava et al. (1989) realizaron la remoción del cromo (III) mediante electrodiálisis, sin embargo, el alcance de sus pruebas sólo se limitó al uso de reactivos químicamente puros. En la industria de curtiembre en el Perú, no se aplica la electrodiálisis como técnica de remoción de iones cromo de sus efluentes.

El objetivo de este trabajo fue remover el cromo (VI) y cromo total de un efluente de curtiembre, para ello, simuló un efluente utilizando una solución acuosa sintética de sulfato básico de cromo de grado industrial. La eficiencia del proceso de electrodiálisis se determinó mediante el porcentaje de extracción y consumo de energía específica para diferentes valores de corriente.

\section{METODOLOGÍA EXPERIMENTAL}

\section{Caracterización del efluente}

Se tomó una muestra del efluente de la zona de curtido en una planta piloto de curtiembre para determinar la concentración de cromo (VI) y cromo total. Los resultados obtenidos fueron: cromo (VI) igual a $18.4 \mathrm{mg} / \mathrm{L}$ y cromo total igual a $251.27 \mathrm{mg} / \mathrm{L}$. A partir de estos resultados, se preparó una solución acuosa sintética con una sal de sulfato básico de cromo de grado industrial.

\section{Módulo de electrodiálisis}

La Figura 1 ilustra el módulo de electrodiálisis utilizado, el cual consistió de una celda de electrodiálisis, una fuente de alimentación de corriente continua (máx. $30 \mathrm{~V}$, máx. $5 \mathrm{~A}$ ), tres bombas (máx. 1400 $\mathrm{mL} / \mathrm{min}$ ) y tres recipientes $(1.3 \mathrm{~L}$ ) para contener las soluciones de lavado de electrodos, concentrado y diluido.

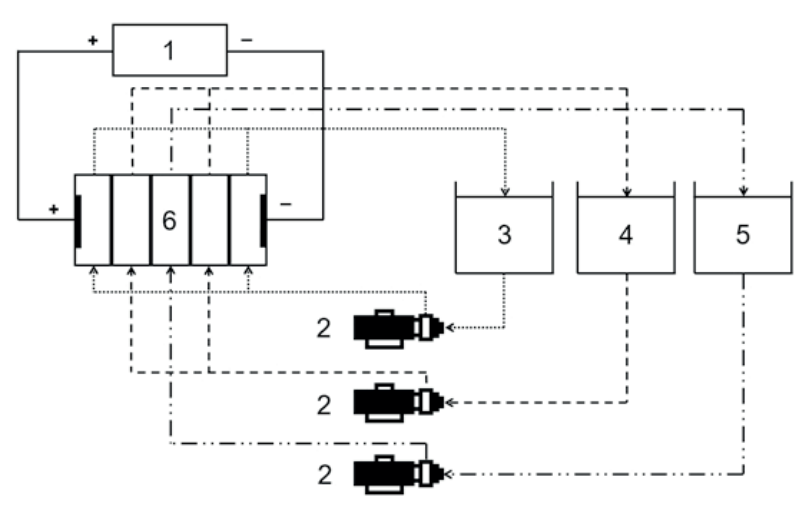

Figura 1. Esquema del módulo de electrodiálisis. 1: Fuente de alimentación de corriente continua; 2 : Bomba; 3, 4 y 5: Recipientes de soluciones de lavado de electrodos, concentrado y diluido, respectivamente; 6 : Celda de electrodiálisis.

Fuente: Elaboración propia

La Figura 2 muestra la celda de electrodiálisis, que consistió de: cuatro membranas de intercambio iónico, dos catiónicas (fumasep FKB-PK-130) y dos aniónicas (fumasep FAB-PK-130), dispuestas en forma alternada de acuerdo a la configuración MC-MAMC-MA, cada una con área de trabajo de $16 \mathrm{~cm}^{2}$; dos electrodos de titanio tipo MMO (óxidos metálicos mezclados) que actuaron como ánodo y cátodo; dos alambres de platino colocados en cada lado de las membranas MA y MC, ubicados en el centro de la configuración para medir el voltaje. Las propiedades físicas y químicas de las membranas de intercambio catiónico y aniónico se indican en la Tabla 1. 


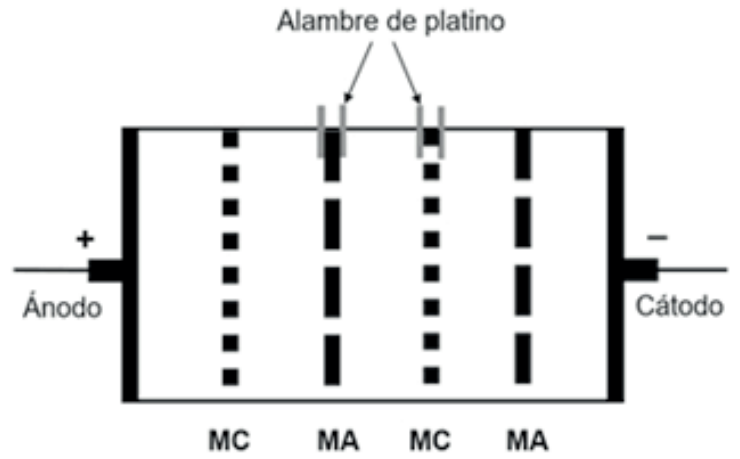

Figura 2. Esquema de la celda de electrodiálisis con tres compartimientos. MC y MA: membranas de intercambio catiónico y aniónico, respectivamente.

Fuente: Elaboración propia

Tabla 1. Propiedades físicas y químicas de las membranas de intercambio iónico.

\begin{tabular}{|l|c|c|}
\hline \multicolumn{1}{|c|}{ Membrana fumasep } & FKB-PK-130 & FAB-PK-130 \\
\hline Tipo de membrana & $\begin{array}{c}\text { Membrana de } \\
\text { intercambio } \\
\text { catiónico }\end{array}$ & $\begin{array}{c}\text { Membrana de } \\
\text { intercambio } \\
\text { aniónico }\end{array}$ \\
\hline Espesor (seco), $\mu \mathrm{m}$ & $126-140$ & $122-133$ \\
\hline $\begin{array}{l}\text { Capacidad de intercambio } \\
\text { iónico, meq.g }\end{array}$ & 0.9 & 0.8 \\
\hline $\begin{array}{l}\text { Conductividad específica, } \\
\text { mS.cm }{ }^{-1}\end{array}$ & 4.0 & 3.6 \\
\hline Resistencia al área, $\Omega . \mathrm{cm}^{2}$ & 3.27 & 3.51 \\
\hline Selectividad, \% & 98 & $>95$ \\
\hline
\end{tabular}

Fuente: Thecnical Data Sheet - fumasep FKB-PK-130 and FABPK-130.

Se prepararon dos litros de solución acuosa de sulfato básico de cromo con una concentración de $2620 \mathrm{mg} / \mathrm{L}$, luego se vertió un litro de la solución preparada en los recipientes de diluido y concentrado, aparte, se preparó un litro de solución de sulfato de sodio con una concentración de $3551 \mathrm{mg} / \mathrm{L}$, el cual se vertió en el recipiente de solución de lavado de electrodos.

Se encendieron las bombas para hacer circular las soluciones por el sistema hidráulico y se midieron los flujos volumétricos del diluido y concentrado, iguales a $1400 \mathrm{~mL} / \mathrm{min}$ y $1053 \mathrm{~mL} / \mathrm{min}$, respectivamente.

\section{Determinación de la corriente límite}

Se hicieron circular las soluciones a través del módulo de electrodiálisis por 3 minutos. Luego se encendió la fuente de alimentación y se fijó un valor de voltaje, a partir de ese momento se tomó el tiempo por 2 minutos para que se estabilice el sistema. Posteriormente, se registraron los valores de corriente en la celda de electrodiálisis y de voltaje en las dos membranas de intercambio catiónico y aniónico. Finalmente, se apagó la fuente de alimentación por 3 minutos y se encendió nuevamente para otro valor de voltaje mayor. Esto se repite hasta que el voltaje de la fuente de alimentación no pase de los $30 \mathrm{~V}$. La Figura 3 muestra la disposición de la fuente de alimentación y los multímetros en la celda de electrodiálisis para determinar la corriente límite.

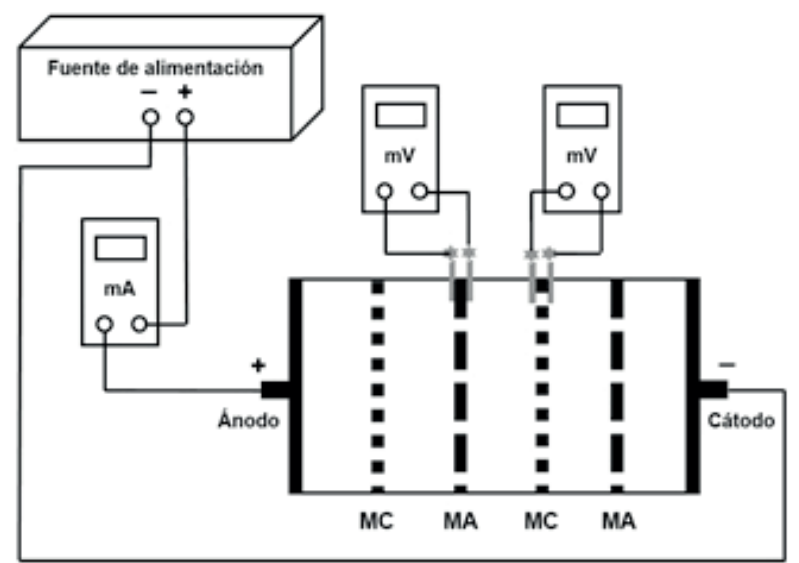

Figura 3. Esquema de la celda de electrodiálisis para determinar la corriente límite.

Fuente: Elaboración propia

\section{Pruebas de electrodiálisis}

Para las pruebas de electrodiálisis se encendió la fuente de alimentación para un voltaje fijo. Cada 2 horas se registró la corriente a través de la celda, la conductividad y el $\mathrm{pH}$ de las soluciones del diluido y concentrado. Cada prueba se realizó hasta que el $\mathrm{pH}$ del diluido no sobrepase el valor de 5.5.

\section{RESULTADOS Y DISCUSIÓN}

\section{Curvas resistencia - inversa de la corriente para las membranas}

Se utilizó el método propuesto por Ponce De León y Field (2000) para determinar la corriente límite, tanto para la membrana de intercambio catiónico como aniónico. Los datos obtenidos de voltaje y corriente de cada membrana fueron representados gráficamente, colocando en la abscisa la inversa de la corriente y en la ordenada la resistencia.

En la Figura 4 para membrana de intercambio catiónico se observa un máximo local $\left(16.67 \mathrm{~A}^{-1}, 11.37\right.$ $\Omega$ ) y mínimo local $\left(70.42 \mathrm{~A}^{-1}, 10.99 \Omega\right)$. En la Figura 5 para membrana de intercambio aniónico se observa un mínimo local (19.92 $\left.\mathrm{A}^{-1}, 13.37 \Omega\right)$. 


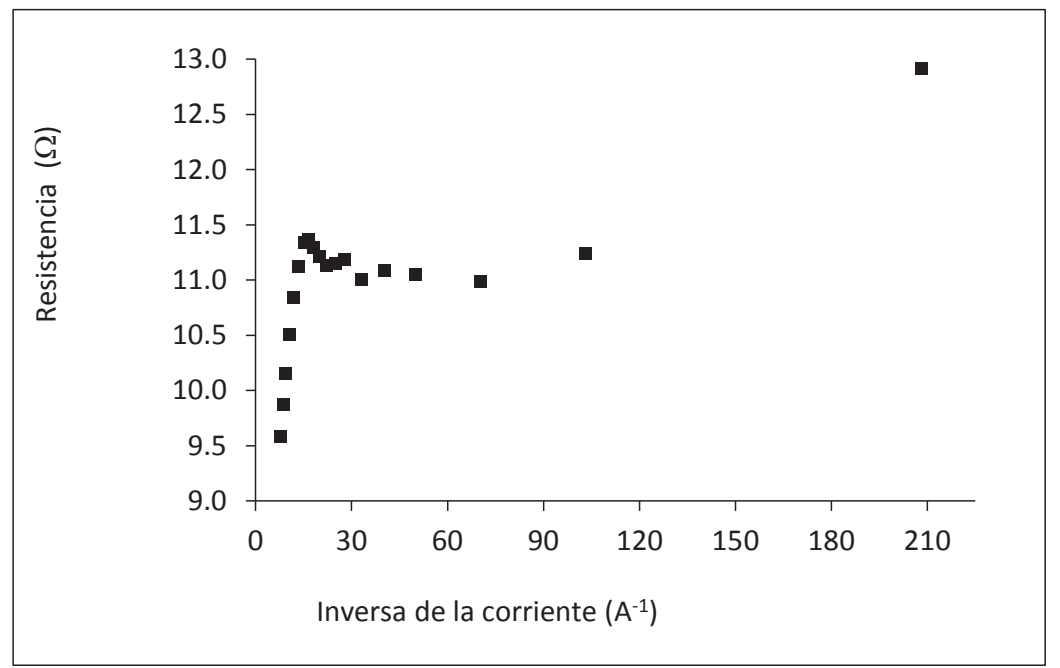

Figura 4. Curva inversa de la corriente - resistencia para la membrana de intercambio catiónico.

Fuente: Elaboración propia

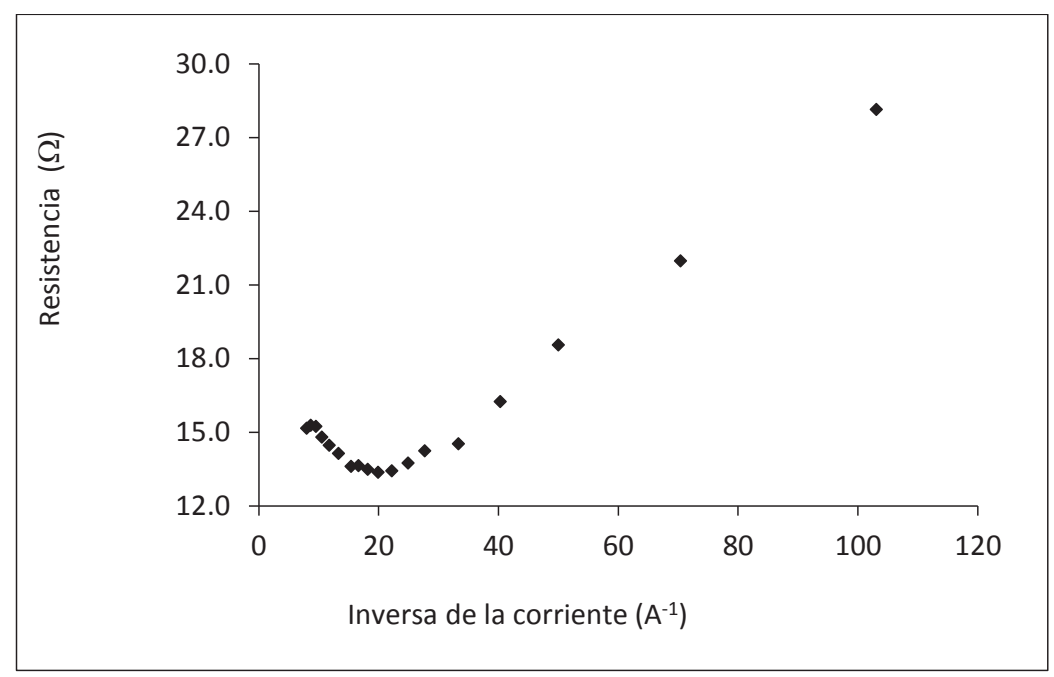

Figura 5. Curva inversa de la corriente - resistencia para la membrana de intercambio aniónico.

Fuente: Elaboración propia

La corriente límite de la celda de electrodiálisis queda determinada por el menor valor local promedio entre la membrana de intercambio catiónico y aniónico (Scarazzato et al., 2015).

En la Tabla 2 se presentan los valores mínimos, máximos y promedios locales de corriente límite para las membranas de intercambio catiónico y aniónico. El valor promedio local más bajo correspondió a la membrana de intercambio catiónico y es $0.0371 \mathrm{~A}$. Para un área de trabajo de membrana de $16 \mathrm{~cm}^{2}$; la densidad de corriente límite fue de $2.32 \mathrm{~mA} / \mathrm{cm}^{2}$.

Tabla 2. Valores mínimos, máximos y promedios locales de corriente límite para las membranas de intercambio iónico.

\begin{tabular}{|c|c|c|c|c|c|}
\hline \multirow{2}{*}{$\begin{array}{c}\text { Membrana de } \\
\text { intercambio iónico }\end{array}$} & \multicolumn{2}{|c|}{ Mínimo local } & \multicolumn{2}{c|}{ Máximo local } & Promedio local \\
\cline { 2 - 6 } & Resistencia $(\Omega)$ & Corriente (A) & Resistencia $(\Omega)$ & Corriente (A) & Corriente límite (A) \\
\hline Catiónico & 10.99 & 0.0142 & 11.37 & 0.0599 & 0.0371 \\
\hline Aniónico & 13.37 & 0.0502 & $>13.37$ & $>0.0502$ & $>0.0502$ \\
\hline
\end{tabular}

Fuente: Elaboración propia 


\section{Curva corriente - voltaje para la celda de elec- trodiálisis}

Los datos obtenidos de voltaje y corriente de la celda de electrodiálisis fueron representados gráficamente, colocando en la abscisa el voltaje y en la ordenada la corriente, obteniéndose la curva de corriente - voltaje. Al proyectar el valor de la corriente límite se obtuvo el voltaje aplicado a la celda de electrodiálisis para la prueba, Figura 6. Para este estudio se asignaron los voltajes al 80,100 y $120 \%$ de la corriente limite (Zhan, 2015). Los valores de los voltajes aplicados se indican en la Tabla 3.

\section{Curvas de $\mathrm{pH}$ y corriente del proceso de elec- trodiálisis}

Para cada prueba de electrodiálisis se realizó mediciones del $\mathrm{pH}$ en el diluido y concentrado cada dos horas (Figura 7 y Figura 8). El pH se trabajó entre los valores de 1 y 5.5 . Si el $\mathrm{pH}<1$, la membrana de intercambio catiónico no es estable para el proceso. $\mathrm{Si}$ el $\mathrm{pH}>5.5$ podría darse la formación de cromo (VI) el cual es tóxico por ser cancerígeno (Chávez, 2010).

También se realizaron las mediciones de corriente a través de la celda de electrodiálisis cada dos horas.

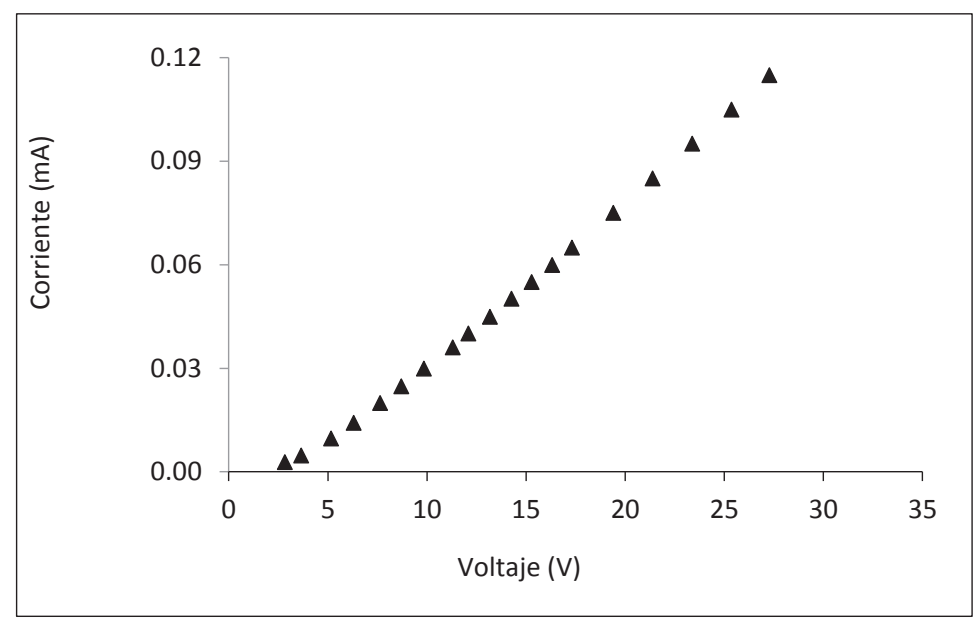

Figura 6. Curva corriente-voltaje para la celda de electrodiálisis.

Fuente: Elaboración propia

Tabla 3. Corriente y voltaje para las pruebas de electrodiálisis.

\begin{tabular}{|c|c|c|}
\hline$\% \mathrm{I}_{\text {lim. }}$ & Corriente (A) & Voltaje (V) \\
\hline 80 & 0.0297 & 10.01 \\
\hline 100 & 0.0371 & 11.65 \\
\hline 120 & 0.0445 & 13.21 \\
\hline \multicolumn{2}{|c|}{ Fuente: Elaboración propia } \\
\hline
\end{tabular}

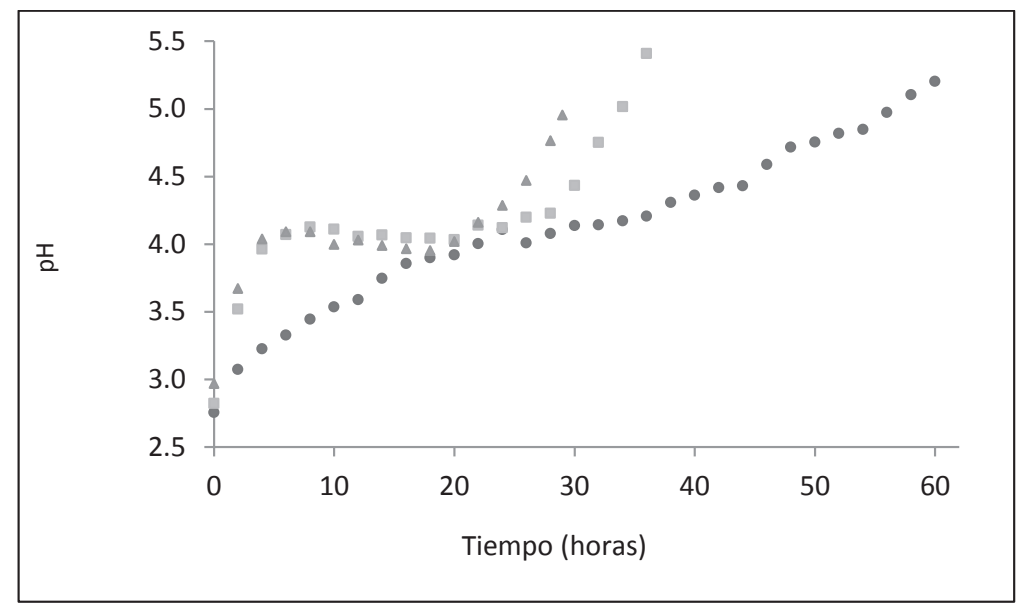

Figura 7. Gráfica del pH del diluido para el voltaje al $80 \%(\bullet), 100 \%(\bullet)$ y $120 \%$ ( $\mathbf{\Delta}$ ) de la corriente límite.

Fuente: Elaboración propia 


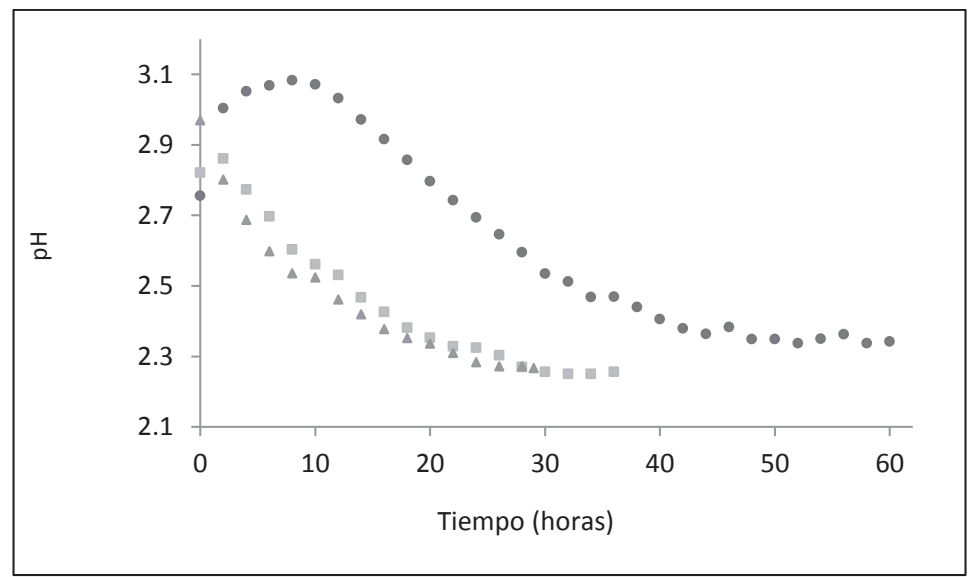

Figura 8. Gráfica del pH del concentrado para la corriente al $80 \%(\bullet), 100 \%(\boldsymbol{\bullet})$ y $120 \%$ ( $\mathbf{\Delta}$ ) de la corriente límite. Fuente: Elaboración propia

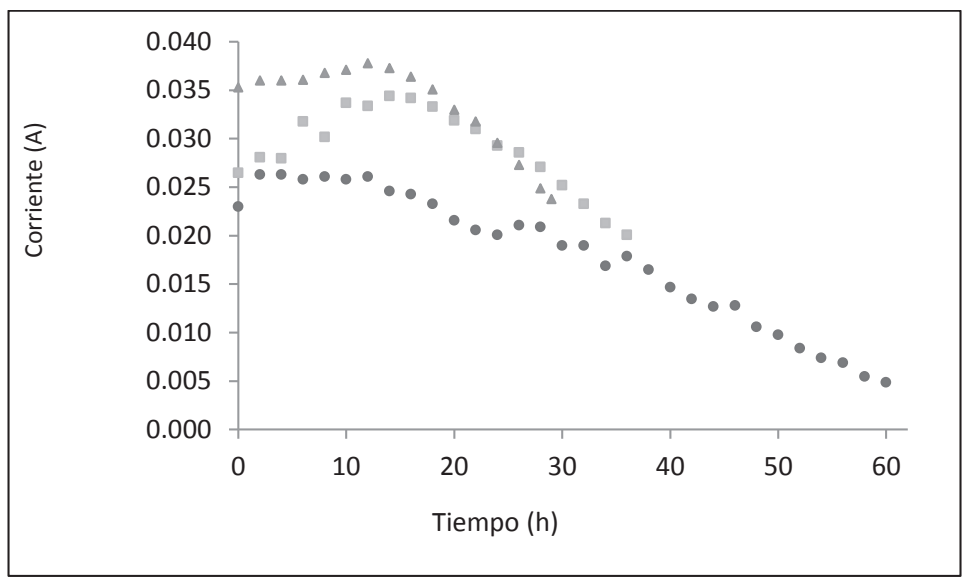

Figura 9. Gráfica de la corriente en la celda para el voltaje al $80 \%(\bullet), 100 \%(\boldsymbol{\bullet})$ y $120 \%$ ( $\boldsymbol{\Delta}$ ) de la corriente límite.

Fuente: Elaboración propia

Como se puede ver en la Figura 9, en las primeras horas la corriente tiende a aumentar y luego disminuye en cada prueba. La disminución de la corriente con respecto al tiempo se debe al empobrecimiento de iones en el compartimiento del diluido que lleva a un aumento de su resistencia eléctrica.

\section{Porcentaje de extracción (PE)}

Es el grado de remoción de los iones de una solución que ha sido tratado con electrodiálisis para un determinado intervalo de tiempo. Se expresa mediante la ecuación (1)

$$
P E=\left(\frac{C_{o}-C_{f}}{C_{o}}\right) \cdot 100 \text { ec (1) }
$$

donde $\underline{C_{o}}$ y ${ }^{C} f$ son las concentraciones inicial y final de la especie química estudiada, respectivamente. La Tabla 4 indica las concentraciones de cromo de la solución acuosa sintética inicial y final provenien- te del compartimiento de diluido de la celda de electrodiálisis, para cada prueba. Las concentraciones finales de cromo total son significativamente inferiores a la concentración inicial. En ninguna de las pruebas se obtuvo concentraciones finales inferiores de los límites máximos permisibles $(2.5 \mathrm{mg} / \mathrm{L})$ y valores referenciales $(5 \mathrm{mg} / \mathrm{L})$ de la normativa peruana (DS Nº03-2002-PRODUCE).

Tabla 4. Concentración de cromo (VI) y cromo total de la solución acuosa sintética al inicio y final de cada prueba.

\begin{tabular}{|c|c|c|c|}
\hline \multirow{2}{*}{$\begin{array}{c}\text { Solución del compartimiento } \\
\text { de diluido }\end{array}$} & \multicolumn{2}{|c|}{ Concentración (mg/L) } \\
\cline { 2 - 4 } & $\mathbf{C r}(\mathbf{V I})$ & $\mathbf{C r}$ total \\
\hline \multirow{3}{*}{ Final } & Inicial & 0.036 & 401.2 \\
\cline { 2 - 4 } & $80 \% \mathrm{I}_{\lim }$ & $<0.01$ & 7.491 \\
\cline { 2 - 4 } & $100 \% \mathrm{I}_{\lim }$ & $<0.01$ & 12.22 \\
\hline & $120 \% \mathrm{I}_{\lim }$ & 0.012 & 21.11 \\
\hline
\end{tabular}

Fuente: Elaboración propia 
En la Tabla 5 se presenta el porcentaje de extracción del cromo (VI) y cromo total. El tiempo de tratamiento no fue igual para cada prueba porque el $\mathrm{pH}$ del diluido condicionó el proceso, es decir que no sobrepasó el valor de 5.5, para evitar la formación de diferentes hidróxidos de cromo (III), especie química predominante del cromo total. El mayor porcentaje de extracción de cromo (VI) y cromo total se obtuvo para el tiempo de tratamiento de $60 \mathrm{~h}$ y voltaje aplicado correspondiente al $80 \% \mathrm{I}_{\text {lim }}$.

Tabla 5. Porcentaje de extracción del cromo (VI) y cromo total para cada prueba.

\begin{tabular}{|c|c|c|c|c|}
\hline \multirow{2}{*}{$\begin{array}{l}N^{\circ} \text { de } \\
\text { prueba }\end{array}$} & \multirow{2}{*}{$\begin{array}{l}\text { Tiempo de } \\
\text { tratamiento } \\
\text { (h) }\end{array}$} & \multirow{2}{*}{$\begin{array}{c}\text { Voltaje } \\
\text { aplicado }\end{array}$} & \multicolumn{2}{|c|}{$\begin{array}{c}\text { Porcentaje de extracción } \\
(\%)\end{array}$} \\
\hline & & & $\mathrm{Cr}(\mathrm{VI})$ & Cr total \\
\hline 1 & 60 & $80 \% I_{\lim }$ & $>72.22$ & 98.13 \\
\hline 2 & 36 & $100 \% I_{\text {lim }}$ & $>72.22$ & 96.95 \\
\hline 3 & 29 & $120 \% \mathrm{I}_{\mathrm{lim}}$ & 66.66 & 94.73 \\
\hline
\end{tabular}

Fuente: Elaboración propia

\section{Consumo de energía específica (CEE)}

Es la energía necesaria para tratar una unidad de volumen de una solución (Demircioglu et al., 2001), se determina por la ecuación (2)

$$
C E E=\frac{\Delta V \int_{o}^{t} I d t}{V_{s}} \quad \mathrm{ec}(2)
$$

donde $\Delta V$ es el voltaje aplicado a la celda, $I$ i es la intensidad de corriente, $t$ es el tiempo que transcurre el proceso y $V_{S}$ es el volumen de la solución a tratar. Los valores de consumo de energía específica se muestran en la Tabla 6 . El menor consumo de energía específica se obtuvo para el tiempo de tratamiento de $60 \mathrm{~h}$ y voltaje aplicado correspondiente al $80 \% \mathrm{I}_{\text {lim }}$.

Por lo tanto, la corriente óptima para el tratamiento de la solución no debe ser mayor de $80 \% \mathrm{I}_{\text {limm }}$, porque podría ocurrir el fenómeno de polarización, esto es, cuando los iones se transportan más rápido a través de la membrana que los que se transportan del seno de la solución a la superficie de la membrana, y como resultado se obtendría el agotamiento de iones en la interfase solución-membrana. Experimentos realizados con solución de cloruro de sodio se encontró que la corriente óptima de suministro fue entre 60 y $80 \% I_{\text {lim }}$ (Chandramowleeswaran, 2006), lo que confirma que la corriente optima está por debajo de la corriente límite.
Tabla 6. Consumo de energía específica para cada prueba.

\begin{tabular}{|c|c|c|c|}
\hline $\begin{array}{c}\mathbf{N}^{\circ} \text { de } \\
\text { prueba }\end{array}$ & $\begin{array}{c}\text { Tiempo de } \\
\text { tratamiento } \\
\text { (h) }\end{array}$ & $\begin{array}{c}\text { Voltaje apli- } \\
\text { cado }\end{array}$ & $\begin{array}{c}\text { CEE } \\
\text { (W.h/L) }\end{array}$ \\
\hline 1 & 60 & $80 \% \mathrm{I}_{\lim }$ & 10.799 \\
\hline 2 & 36 & $100 \% \mathrm{I}_{\lim }$ & 12.325 \\
\hline 3 & 29 & $120 \% \mathrm{I}_{\lim }$ & 13.020 \\
\hline
\end{tabular}

Fuente: Elaboración propia

\section{CONCLUSIONES}

El estudio demostró que es factible remover el cromo de una sal de grado industrial con electrodiálisis. El proceso es más eficiente para un voltaje aplicado que corresponde al $80 \% \mathrm{I}_{\text {lim }}$, aun cuando el tiempo de tratamiento es mayor. Se recomienda realizar pruebas ajustando el valor de $\mathrm{pH}$, por debajo de 5.5 .

\section{AGRADECIMIENTOS}

Los autores expresan su agradecimiento al Instituto General de Investigación de la Universidad Nacional de Ingeniería, así como a la Dra. Tatiane Benvenuti del Laboratorio de Corrosión, Protección y Reciclaje de Materiales de la Universidad Federal de Río Grande do Sul, por el apoyo brindado.

\section{REFERENCIAS}

[1] Chandramowleeswaran, M., y Palanivelu, K. (2006). Treatability studies on textile effluent for total disolved solids reduction using electrodialysis. Desalination 201, 164-174.

[2] Chávez, A. (2010). Descripción de la nocividad del cromo proveniente de la industria curtiembre y de las posibles formas de removerlo. Revista Ingenierías Universidad de Medellín, 9 (17), 41-50.

[3] Cowan, D., y Brown, J. (1959). Effect of turbulence on limiting current in electrodialysis cells. Industrial \& Engineering Chemistry, 51 (12), 1445-1448.

[4] Demircioglu, M., Kabay, N., Ersoz, E., Kurucaovali, I., Safak, C., y Gizli, N. (2001). Cost comparison and efficiency modeling in the electrodialysis of brine. Desalination, 136 (Issues 1-3), 317-323.

[5] D.S. No 003-2002-PRODUCE (2002, viernes 4 de octubre). Límites máximos permisibles 
y valores referenciales para las actividades industriales de cemento, cerveza, curtiembre y papel. El Peruano Normas Legales: 230921230925.

[6] D.S. N 021-2009-VIVIENDA (2009, viernes 20 de noviembre). Valores máximos admisibles (VMA) de las descargas de aguas residuales no domésticas en el sistema de alcantarillado sanitario. El Peruano Normas Legales: 406305406307.

[7] Koter, S., y Warszawski, A. (2000). Electromembrane processes in environment protection. Polish Journal of Environmental Studies, 9 (1), 45-56.

[8] Lambert, J., Rakib, M., Durand, G., y AvilaRodriguez, M. (2006). Treatment of solutions containing trivalent chromium by electrodialysis. Desalination, 191 (Issues 1-3), 100-110.

[9] Martí, M. (2014). Estudio del transporte de iones de metales pesados a través de membranas de intercambio catiónico aplicado al tratamiento de efluentes industriales (Tesis doctoral). Universidad Politécnica de Valencia, Valencia, España.

[10] Ochoa, J. (1996). Electrosíntesis y electrodiálisis: Fundamentos, aplicaciones tecnológicas y tendencias. Madrid, España: McGraw-Hill.
[11] Ponce de León, C., y Field, R. (2000), On the determination of limiting current density from uncertain data. Journal of Applied Electrochemistry, 30, 1087-1090.

[12] Praneeth, K., Manjunath, D., Bhargava, S., Tardio, J., y Shidhar, S. (2014). Economical treatment of reverse osmosis reject of textile industry effluent by electrodialysis-evaporation integrated process. Desalination, 333 (1), 82-91.

[13] Raghava, J., Prasad, B., Narasimham, V., Ramasami, T., Shah, P., y Khan, A. (1989), Electrodialysis in the recovery and reuse of chromium from industrial effluents. Journal of Membrane Science, 46 (Issues 2-3), 215-224.

[14] Scarazzato, T., Buzzi, D., Bernardes, A., Tenório, J., y Espinosa, D. (2015). Currentvoltage curves for treating effluent containing HEDP: Determination of the limiting current. Brazilian Journal of Chemical Engineering, 32 (4), 831-836.

[15] Strathmann, H. (2010). Electrodialysis, a mature technology with a multitude of new applications. Desalination, 264, 268-288.

[16] Zhan, Z. (2015). Tratamiento de aguas residuales y evaluación de la calidad del agua a través de enfoques electroquímicos (Tesis de maestría). Universidad de Lakehead, Ontario, Canada. 